\title{
Aporte antioxidante del plasma seminal y su efecto sobre la calidad del semen equino congelado
}

\author{
Antioxidant contribution of seminal plasma and its effect on the quality \\ of frozen equine semen
}

\author{
Giovanni Restrepo B. ${ }^{1,4}$, Edison Pizarro L. ${ }^{2}$, Benjamín A. Rojano ${ }^{3}$
}

\section{Resumen}

\begin{abstract}
El plasma seminal (PS) posee antioxidantes enzimáticos y no enzimáticos con la función de proteger los espermatozoides de los efectos nocivos del estrés oxidativo. El objetivo de este estudio fue evaluar el aporte antioxidante del PS durante la congelación del semen equino y su efecto sobre la calidad espermática posdescongelación. Se colectó el semen de cinco caballos, se separó el PS y se suplementó en proporciones del $0 \%$ (Control), 10\% (PS10) y 20\% (PS20) a la fracción espermática en el diluyente de congelación. Se evaluó la capacidad antioxidante total (CAT) por los métodos de la capacidad atrapadora de radical oxígeno (ORAC) y de la capacidad reductora férrica (FRAP), y la actividad de las enzimas SOD, GPx y catalasa mediante espectrofotometría. Quince eyaculados fueron sometidos a congelación y se evaluó la movilidad (MOV), vitalidad (VE), morfología (MA), integridad funcional de membrana (IM) y el potencial de membrana mitocondrial (PMM) de los espermatozoides. Se ajustaron modelos mixtos, se compararon las medias por la prueba de Tukey y se realizó un análisis de correlación. PS10 y PS20 generaron un incremento de la CAT medida por FRAP y de la actividad de catalasa del semen diluido $(\mathrm{p}<0.05)$. PS20 produjo un aumento de la MOV del semen descongelado en comparación con el Control $(\mathrm{p}<0.05)$. Se observó una reducción posdescongelación del PMM por efecto de PS10 y PS20 ( $<<0.05)$, y se hallaron correlaciones positivas entre el PMM y la actividad de SOD. Se concluye que el PS aumenta la capacidad antioxidante total y enzimática del semen equino e influye en la actividad mitocondrial y la movilidad espermática posdescongelación.
\end{abstract}

Palabras clave: antioxidantes; enzimas; criopreservación; diluyente; semen

\footnotetext{
${ }^{1}$ Facultad de Ciencias Agrarias, Universidad Nacional de Colombia, Medellín, Colombia

${ }^{2}$ Facultad de Ciencias Agrarias, Politécnico Colombiano Jaime Isaza Cadavid, Medellín, Colombia

${ }^{3}$ Facultad de Ciencias, Universidad Nacional de Colombia, Medellín, Colombia

${ }^{4}$ E-mail: grestre0@unal.edu.co
}

Recibido: 11 de julio de 2018

Aceptado para publicación: 23 de noviembre de 2018 
The seminal plasma (SP) has enzymatic and non-enzymatic antioxidants with the function of protecting sperm from the harmful effects of oxidative stress. The aim of this study was to evaluate the antioxidant contribution of SP during the freezing of equine semen and its effect on post-thawing sperm quality. The semen of five horses was collected, the SP was separated and supplemented in proportions of $0 \%$ (Control), $10 \%$ (PS10) and 20\% (PS20) to the sperm fraction in the freezing extender. The total antioxidant capacity (TAC) was evaluated by the oxygen radical trapping capacity (ORAC) and ferric reducing capacity (FRAP) methods, and the activity of SOD, GPx and catalase enzymes by spectrophotometry. Fifteen ejaculates were subjected to freezing and motility (MOT), vitality (EV), morphology (AM), functional membrane integrity (MI) and mitochondrial membrane potential (MMP) of the sperm were evaluated. Mixed models were adjusted, the means were compared by the Tukey test and a correlation analysis was performed. PS10 and PS20 generated an increase in the TAC measured by FRAP and the catalase activity of the diluted semen $(\mathrm{p}<0.05)$. PS20 increased the MOT of thawed semen compared to the Control $(\mathrm{p}<0.05)$. A post-thawing reduction of MMP was observed due to PS10 and PS20 $(\mathrm{p}<0.05)$, and positive correlations were found between MMP and SOD activity. It is concluded that SP increases the total and enzymatic antioxidant capacity of stallion semen and influences mitochondrial activity and post-thaw sperm motility.

Key words: antioxidants; enzymes; cryopreservation; extender; semen

\section{INTRODUCCIÓN}

La congelación del semen equino produce un incremento en la generación de especies reactivas de oxígeno (ERO) debido al desacoplamiento del metabolismo oxidativo normal de los espermatozoides (OrtegaFerrusola et al., 2009). Esto se explica por la pérdida de la actividad de defensa antioxidante (Isachenko et al., 2004), la presencia de células apoptóticas (Brum et al., 2008) y la centrifugación del semen durante su procesamiento (Vasconcelos et al., 2010). El estrés oxidativo desencadena la oxidación de los ácidos grasos, de las proteínas e incluso del ADN de los espermatozoides equinos (Morte et al., 2008), lo cual genera una reducción de la movilidad, la vitalidad y la capacidad de dichas células para la fertilización (Baumber et al., 2002).
Otro proceso que favorece el estrés oxidativo del semen equino criopreservado es la remoción del plasma seminal (PS), explicado por su posible relación con la incapacidad de los espermatozoides para sufrir reacción acrosómica, unirse a la zona pelúcida y completar la fertilización (Ferrante et al., 2016; Al-Essawe et al., 2018b); así como por otros efectos deletéreos sobre la calidad y la vitalidad espermática (Contri et al., 2012; Ramires-Neto et al., 2013a). Por otro lado, se sabe que el PS provee la mayor parte de los antioxidantes del semen equino (Moore et al., 2005; Wnuk et al., 2010), dado que, entre su gran diversidad de componentes, posee antioxidantes enzimáticos y no enzimáticos, los cuales tendrían la función de proteger los espermatozoides de los efectos nocivos del estrés oxidativo (Guasti et al., 2012; Waheed et al., 2013; Bucci et al., 2016). 
El PS de los equinos está involucrado en eventos como la activación de la movilidad espermática, la acción antimicrobiana, la neutralización de metabolitos, la capacitación espermática y la respuesta inflamatoria uterina poscoital (Kareskoski y Katila, 2008; Rodríguez-Martínez et al., 2011; Boe-Hansen et al., 2015). Además, es considerado como esencial en la calidad seminal, en el proceso de fecundación y en la congelabilidad del semen equino (Ramires-Neto et al., 2013b; Bucci et al., 2016; Usuga et al., 2018).

La adición de diferentes proporciones de PS a los diluyentes para la congelación de semen equino se ha considerado como una alternativa para el mejoramiento de la calidad espermática posdescongelación, a través de la reducción del estrés oxidativo (Guasti et al., 2012; Pizarro et al., 2013; Waheed et al., 2013). Sin embargo, se desconoce en gran medida el aporte que el PS realiza a la capacidad antioxidante total (CAT) y a la actividad enzimática del semen, y como esto podría relacionarse con la reducción de los efectos deletéreos de la criopreservación sobre los espermatozoides. El objetivo de este estudio fue evaluar el aporte antioxidante del PS durante la congelación del semen equino y su efecto sobre la calidad espermática posdescongelación.

\section{Materiales y Métodos}

\section{Muestras Seminales}

Se utilizaron cinco caballos criollos colombianos (Equus caballus) ubicados en el norte del Valle de Aburra, departamento de Antioquia (Colombia). La zona presenta una altitud promedio de $1475 \mathrm{msnm}$, temperatura promedio de $22{ }^{\circ} \mathrm{C}$ y pluviosidad cercana a $\operatorname{los} 2200 \mathrm{~mm} /$ año.

Los animales tenían entre 2 y 8 años y las colectas se hicieron en un régimen de una colecta semanal como mínimo. Los caballos tenían una condición corporal de 6-7 (escala entre 1 y 9), fertilidad comprobada mediante crías nacidas vivas y estuvieron sometidos a condiciones iguales de manejo en estabulación, con alimentación a base de pasto estrella henificado (Cynodon nlemfuensis) suministrado a voluntad.

Se colectaron 15 eyaculados mediante el método de la vagina artificial, con una vagina modelo Missouri (Minitube, Tiefenbach, Alemania), lubricada con gel no espermicida y sobre una yegua. La fracción en gel del eyaculado fue removida por filtración. El semen se diluyó en proporción 1:1 en diluyente EquiPlus $^{\circledR}$ (Minitube, Tiefenbach, Alemania). Con la finalidad de obtener el PS, se centrifugaron $20 \mathrm{ml}$ de cada eyaculado a 800 $g$ por 15 minutos (Ultra-8V, LW Scientific). Tanto el semen diluido como el PS se llevaron al laboratorio en condiciones de refrigeración en una caja de Neopor (Minitube, Tiefenbach, Alemania).

\section{Criopreservación del Semen}

La criopreservación se realizó mediante un protocolo de congelación modificado del reportado por Bustamante et al. (2009). El semen diluido se centrifugó a $1200 \mathrm{~g}$ por 15 $\min$ (Mikro 220R, Hettich, Alemania). El precipitado se resuspendió en un diluyente de congelación a base de leche semidescremada, caseinatos de sodio y azúcares, suplementado con $4 \%$ de yema de huevo y $5 \%$ de $N, N$ dimetilformamida (Sigma-Aldrich), hasta alcanzar una concentración final de $100 \times 10^{6}$ espermatozoides $/ \mathrm{ml}$. El semen se dividió en tres alícuotas, las cuales fueron asignadas aleatoriamente a uno de tres tratamientos: $0 \%$ de PS (control), $10 \%$ de PS (PS10), 20\% de PS (PS20). La suplementación se realizó con PS autólogo de cada caballo incluido en el estudio, en procesos independientes de congelación seminal. Posteriormente, el semen diluido se mantuvo a $5{ }^{\circ} \mathrm{C}$ por 15 min y luego se empacó manualmente en pajillas de 0.5 $\mathrm{ml}$. Las pajillas se sometieron a vapores de nitrógeno líquido por $15 \mathrm{~min}$, para finalmente ser almacenadas en un termo con nitrógeno líquido. Después de un periodo de almacena- 
miento mínimo de dos semanas, las pajillas de semen se descongelaron en agua a $37^{\circ} \mathrm{C}$ por $1 \mathrm{~min}$.

\section{Capacidad Antioxidante Total (CAT)}

Antes de la congelación, se evaluó la CAT del semen diluido y suplementado con diferentes proporciones de PS. Se emplearon los ensayos de la capacidad atrapadora de radical oxígeno (ORAC) y de la capacidad reductora férrica (FRAP).

El método ORAC estuvo basado en reportes de Ou et al. (2001). Se utilizaron soluciones de fluoresceína $1 \times 10^{-5} \mathrm{M}$ en PBS (75 mM, pH 7.4) y de AAPH 0.6 M en PBS (75 mM, pH 7.4). Cada tubo de reacción se preparó con $21 \mu \mathrm{l}$ de fluoresceína, $2.899 \mu \mathrm{l}$ de PBS, $30 \mu 1$ de muestra y $50 \mu 1$ de AAPH. Se emplearon condiciones controladas de temperatura $37^{\circ} \mathrm{C}, \mathrm{pH} 7.4$ y Trolox ${ }^{\circledR}$ (Merck, Darmstadt, Alemania) como antioxidante de referencia. Las lecturas se realizaron en un espectrofluorímetro LS 55 (Perkin Elmer, Waltham, USA) a longitudes de excitación/ emisión de 493/515 nm. El valor ORAC se calculó usando las diferencias de áreas bajo la curva de decaimiento de la fluoresceína entre un blanco y la muestra, lo cual se comparó contra una curva patrón con Trolox ${ }^{\circledR}$.

Para el método FRAP se adicionaron $50 \mu 1$ de muestra a $900 \mu 1$ de solución FRAP compuesta por buffer ácido acético-acetato de sodio (pH 3.4), TPTZ y $\mathrm{FeCl}_{3}$, en relación 10:1:1. Luego de 30 min de reacción se midió la absorbancia a una longitud de onda de $593 \mathrm{~nm}$ en un espectrofotómetro $6405 \mathrm{UV} /$ Vis (Jenway, Burlington, USA). El valor encontrado se comparó con una curva de referencia construida con ácido ascórbico (Benzie y Strain, 1996).

\section{Actividad Antioxidante Enzimática}

La actividad del glutatión peroxidasa (GPx) y la superóxido dismutasa (SOD) del semen diluido y suplementado con diferentes proporciones de PS se midió mediante los kits Ranse ${ }^{\circledR}$ y Ransod ${ }^{\circledR}$ (Randox Laboratories, Crumlin, UK), respectivamente, de acuerdo a los procedimientos descritos por Tavilani et al. (2008). Para la GPx se preparó una solución de reacción con glutatión (4 $\mathrm{mmol} / \mathrm{l})$, NADPH $(0.34 \mathrm{mmol} / \mathrm{l})$ y glutatión reductasa $(0.5 \mathrm{U} / \mathrm{l})$. Dicha solución se mezcló con hidroperóxido de cumeno $(0.18 \mathrm{mmol} /$ 1) y $0.05 \mathrm{ml}$ de muestra. Se midió el descenso en la absorbancia por espectrofotometría a $340 \mathrm{~nm}$. Para la SOD la muestra se diluyó en proporción 1:31 en buffer fosfato $(10 \mathrm{mM}$, pH 7). Se empleó xantina $(0.05 \mathrm{mmol} / \mathrm{l})$ y la enzima xantina oxidasa $(80 \mathrm{U} / \mathrm{l})$ para generar radical superóxido, el cual reaccionó con INT (2-(4-yodofenol)-3-(4-nitrofenol)-5-fenil tetrazolium cloruro) $(0.025 \mathrm{mmol} / \mathrm{l})$, para generar formazán. Se definió una unidad de SOD como aquella que inhibió el $50 \%$ de la producción de formazán. Las lecturas se realizaron por espectrofotometría a $505 \mathrm{~nm}$.

Se evaluó la actividad de catalasa del semen diluido y suplementado con PS mediante la medición de la desaparición de peróxido de hidrógeno, de acuerdo con el procedimiento descrito por Aebi (1984). Se mezclaron $30 \mu \mathrm{l}$ de muestra con $720 \mu \mathrm{l}$ de una solución de peróxido compuesta por buffer PBS (10 mM, pH 7.0), Tritón X-100 (0.1\%) y peróxido de hidrógeno $(20 \mathrm{mM})$. Luego se midió el descenso de la absorbancia por espectrofotometría a $240 \mathrm{~nm}$, durante $3 \mathrm{~min}$. Una unidad de catalasa se definió como $1 \mu$ mol de peróxido de hidrógeno consumido por minuto. Las lecturas para las tres enzimas se realizaron mediante un espectrofotómetro 6405 UV/Vis (Jenway, Burlington, USA).

\section{Calidad Seminal}

- El volumen de semen libre de gel de cada eyaculado se midió mediante un tubo graduado.

- La concentración de espermatozoides se evaluó a partir de una gota de semen fresco mediante espectrofotometría (Spermacue $^{\circledR}$, Minitube, Tiefenbach, Alemania). 
Cuadro 1. Capacidad antioxidante total y enzimática de semen equino diluido y suplementado con dos proporciones de PS ( $\mathrm{n}=15)$

\begin{tabular}{lccccc}
\hline Tratamiento & ORAC & FRAP & SOD & GPx & Catalasa \\
\hline Control & $7283.1 \pm 3.5^{\mathrm{a}}$ & $24.0 \pm 10.6^{\mathrm{b}}$ & $2.3 \pm 1.5^{\mathrm{a}}$ & $275.3 \pm 204.7^{\mathrm{a}}$ & $83.5 \pm 46.5^{\mathrm{b}}$ \\
P10 & $7088.0 \pm 1.6^{\mathrm{a}}$ & $37.2 \pm 6.5^{\mathrm{a}}$ & $2.3 \pm 1.9^{\mathrm{a}}$ & $318.0 \pm 161.6^{\mathrm{a}}$ & $126.6 \pm 31.0^{\mathrm{a}}$ \\
P20 & $6524.7 \pm 2.5^{\mathrm{a}}$ & $40.6 \pm 6.5^{\mathrm{a}}$ & $3.4 \pm 1.8^{\mathrm{a}}$ & $376.4 \pm 160.6^{\mathrm{a}}$ & $168.3 \pm 63.9^{\mathrm{a}}$ \\
\hline
\end{tabular}

Los resultados se expresan como media \pm desviación estándar.

P10: suplementación con $10 \%$ de plasma seminal; P20: suplementación con $20 \%$ de plasma seminal

ORAC: $\mu \mathrm{mol}$ trolox equivalente/I; FRAP: $\mathrm{mg}$ de ácido ascórbico equivalente/l; SOD, GPx y Catalasa: unidades de enzima/ml

Letras diferentes indican diferencia estadística significativa entre tratamientos $(p<0.05)$

- La movilidad espermática (MOV) se evaluó por microscopía de contraste de fase (Eclipse E200 ${ }^{\circledR}$, Nikon Inc., Tokio, Japón) en cinco campos de observación como mínimo.

- La vitalidad espermática(VE) y la morfología anormal (MA) se evaluaron por la técnica de eosina-nigrosina modificada (Brito et al., 2011), para lo cual, sobre un portaobjetos se mezclaron una gota de semen y una gota de eosinanigrosina (Sigma-Aldrich, St. Louis, USA) y se realizó un extendido. En un microscopio de contraste de fase Eclipse E200 (Nikon Inc., Tokio, Japón), se realizó la evaluación individual de 200 espermatozoides.

- La integridad funcional de la membrana plasmática (IM) de los espermatozoides se evaluó por la prueba hipoosmótica descrita por Neild et al. (1999). En un tubo se mezclaron $100 \mu 1$ de semen con 500 $\mu 1$ de solución hipoosmótica de sacarosa 5.4\% (100 mOsmol/1). Dicha mezcla se incubó a $38.5^{\circ} \mathrm{C}$ por $30 \mathrm{~min}$ y se evaluaron 200 espermatozoides, mediante un microscopio de contraste de fase Eclipse E200 (Nikon Inc. Tokio, Japón).

- El potencial de membrana interna mitocondrial (PMM) de los espermato- zoides se evaluó mediante la sonda fluorescente catiónica JC-1 (Molecular Probes $^{\mathrm{TM}}$, Waltham, USA) (Gravance $e t$ al., 2000). El semen se diluyó hasta $20 \mathrm{x}$ $10^{6}$ espermatozoides $/ \mathrm{ml}$, se incubó por 20 min a $35^{\circ} \mathrm{C}$ con JC- 1 en DMSO ( 2 $\mathrm{mM})$. Las lecturas se realizaron mediante un espectrofluorímetro LS 55 (Perkin Elmer, Waltham, USA) a longitudes de excitación/emisión de 514/529 nm para PMM bajos (JC-1 monómeros, fluorescencia verde) y de 585/590 nm para PMM altos (JC-1 agregados, fluorescencia naranja).

\section{Análisis Estadístico}

Se ajustaron modelos mixtos donde se incluyó el efecto fijo del tratamiento, el efecto de la repetición y el efecto aleatorio anidado del eyaculado dentro del equino. Con la prueba de Shapiro-Wilk se comprobó la normalidad de los datos. La comparación de las medias entre los tratamientos se realizó mediante la prueba de Tukey. El nivel de significancia fue $p<0.05$. Se realizó un análisis de correlación de Pearson entre las variables dependientes. Todos los análisis se realizaron mediante el programa SAS 9.2. (SAS Inst. Inc., NC, USA). 
Cuadro 2. Calidad del semen equino fresco ( 5 caballos y 15 eyaculados)

\begin{tabular}{lcccccc}
\hline Variable & Media & DE & CV & EE & Mínimo & Máximo \\
\hline Volumen (ml) & 32.4 & 20.3 & 62.5 & 3.6 & 7 & 85 \\
Concentración & 236.7 & 108.4 & 45.8 & 19.2 & 108 & 550 \\
MOV (\%) & 75.6 & 10.1 & 13.4 & 1.8 & 50 & 90 \\
VE (\%) & 80.7 & 7.9 & 9.8 & 1.4 & 66 & 94 \\
MA (\%) & 17.6 & 6.9 & 39.3 & 1.2 & 6 & 31 \\
IM (\%) & 74.7 & 10.7 & 14.3 & 2.1 & 39 & 87 \\
\hline
\end{tabular}

DE: desviación estándar; CV: coeficiente de variación (\%); EE: error estándar.

MOV: movilidad espermática; VE: vitalidad espermática; MA: morfología anormal: IM: integridad funcional de la membrana plasmática

La concentración es expresada como espermatozoides $\times 10^{6} / \mathrm{ml}$

Cuadro 3. Calidad del semen equino descongelado $\left(n=60^{1}\right)$

\begin{tabular}{lcccc}
\hline Tratamiento & MOV $(\%)$ & VIT $(\%)$ & MA $(\%)$ & IM (\%) \\
\hline Control & $38.1 \pm 13.0^{\mathrm{b}}$ & $53.9 \pm 11.5^{\mathrm{a}}$ & $17.2 \pm 5.3^{\mathrm{a}}$ & $37.4 \pm 10.4^{\mathrm{a}}$ \\
P10 & $39.3 \pm 8.7^{\mathrm{ab}}$ & $53.1 \pm 10.1^{\mathrm{a}}$ & $16.0 \pm 5.3^{\mathrm{a}}$ & $36.1 \pm 8.4^{\mathrm{a}}$ \\
P20 & $41.2 \pm 10.5^{\mathrm{a}}$ & $54.9 \pm 8.8^{\mathrm{a}}$ & $17.2 \pm 5.0^{\mathrm{a}}$ & $37.5 \pm 8.6^{\mathrm{a}}$ \\
\hline
\end{tabular}

${ }^{1}$ Cuatro pajillas de semen por eyaculado y tratamiento

Los resultados se expresan como media \pm desviación estándar

P10: suplementación con $10 \%$ de plasma seminal; P20: suplementación con $20 \%$ de plasma seminal

MOV: movilidad espermática; VIT: vitalidad espermática; MA: morfología anormal; IM: integridad funcional de la membrana plasmática

Letras diferentes indican diferencia estadística significativa entre tratamientos $(p<0.05)$

\section{Resultados y Discusión}

Algunas investigaciones han reportado la CAT del PS equino, evaluada a través de metodologías como la capacidad atrapadora de radicales oxígeno (ORAC), la decoloración del radical catiónico $A B T S^{\mathrm{i} \%^{+}}$(ABTS) y el poder reductor férrico (FRAP) (Wnuk et al., 2010; Restrepo et al., 2015). Así mismo, se ha estimado la CAT del semen equino diluido con fines de congelación, a través de los ensayos ORAC y FRAP (Restrepo y Rojano, 2017). En esta investigación se en- contró que la suplementación con PS en el semen diluido en un medio de congelación genera un incremento de la CAT representada por FRAP; mientras no se observó el mismo efecto sobre la CAT evaluada mediante ORAC (Cuadro 1). No se observó un efecto atribuible a la proporción de PS (10 o 20\%) sobre la CAT; sin embargo, se encontraron niveles de CAT cercanos a un estudio previo con semen equino diluido (Restrepo y Rojano, 2017) y valores superiores a la CAT previamente reportada para el PS equino (Restrepo et al., 2015). De tal forma, que es necesario considerar para el semen diluido, la presen- 
cia de sustancias antioxidantes propias del semen, así como de otras disponibles en el diluyente, las cuales incluso ante la remoción del PS puede ejercer un efecto compensatorio de la actividad antioxidante (Bustamante et al., 2009).

La congelación del semen produce un incremento en la generación de ERO (Ortega-Ferrusola et al., 2009), entre las cuales, se ha descrito al peróxido de hidrógeno como la principal especie causante de citotoxicidad en el semen equino (Burnaugh et al., 2007). Así mismo, se ha reportado que la adición de 10 y $20 \%$ de PS al semen diluido puede generar un incremento en la producción de ERO; sin embargo, por el aporte antioxidante del PS se observó una reducción de la peroxidación lipídica del semen descongelado (Pizarro et al., 2013).

El PS equino posee actividades considerables de SOD y GPx (Baumber et al., 2005, Villa et al., 2012). Así mismo, la catalasa se encuentra en grandes cantidades en todas las fracciones de un eyaculado, a excepción del fluido pre-espermático (Koskinen et al., 2002). Sin embargo, durante la congelación del semen equino se produce una reducción de la actividad enzimática por la inhibición generada por las ERO y por algunos productos tóxicos de la peroxidación lipídica (Baumber et al., 2000), así como por efecto de la remoción del PS (Guasti et al., 2012). En este estudio se observó un incremento de la actividad de catalasa por la adición de PS, sin encontrar un aporte significativo del PS sobre la actividad de SOD y GPx, posiblemente explicado por la alta variabilidad de los resultados (Cuadro 1). La actividad de catalasa y SOD se han relacionado con la disminución del estrés oxidativo y el mejoramiento de la calidad del semen equino (Baumber et al., 2003; Cocchia et al., 2011).

Se observó una importante reducción de los parámetros espermáticos entre el semen fresco (Cuadro 2) por efecto de la criopreservación, incluso con el uso de diferentes proporciones de PS en el semen dilui- do para la congelación (Cuadro 3). La suplementación con $20 \%$ de PS sobre la MOV del semen descongelado fue significativamente diferente en comparación con el control sin PS; efecto que ya había sido previamente descrito para concentraciones del 20 y el 50\% de PS (Neuhauser et al., 2015). Sin embargo, la adición de PS no generó efecto alguno sobre la morfología, la vitalidad o la integridad funcional de la membrana plasmática (Cuadro 3). Esto podría explicarse por la variabilidad en la composición del PS de los reproductores, toda vez que, en estudios previos se observó que diferentes niveles de proteínas, vitaminas y iones presentes en el PS influyen, no solo en la calidad del semen fresco, sino también en la calidad del semen criopreservado cuando se adiciona PS (Usuga et al., 2017, 2018). En un estudio reciente se encontró que la selección espermática mediante centrifugación de una sola capa (SLC) antes de la criopreservación mejoró la calidad espermática posdescongelación, y que la inclusión de PS de caballos «buenos» $\mathrm{y}$ «malos» congeladores no tuvo un efecto benéfico adicional (AlEssawe et al., 2018a).

Entre los marcadores de apoptosis que pueden ser usados para predecir la resistencia a la congelación de los espermatozoides equinos, la actividad mitocondrial tiene el máximo poder diagnóstico (Ortega-Ferrusola et al., 2009). La reducción en la actividad mitocondrial de los espermatozoides por efecto de la criopreservación ha sido ampliamente descrita (Schober et al., 2007). En este estudio se observó una reducción de los niveles de PMM altos (JC-1 naranja, formación de agregados) y de PMM bajos (JC1 verde, monómeros) por la suplementación con PS para la congelación espermática (Figura $1)$. Este efecto podría relacionarse con alteraciones mitocondriales ocasionadas por un incremento en la producción de ERO por la adición de PS (Pizarro et al., 2013). Sin embargo, también podría asociarse a las modificaciones en el estado redox del espermatozoide y posiblemente de sus mitocondrias, por el aporte de diferentes moléculas involucradas 


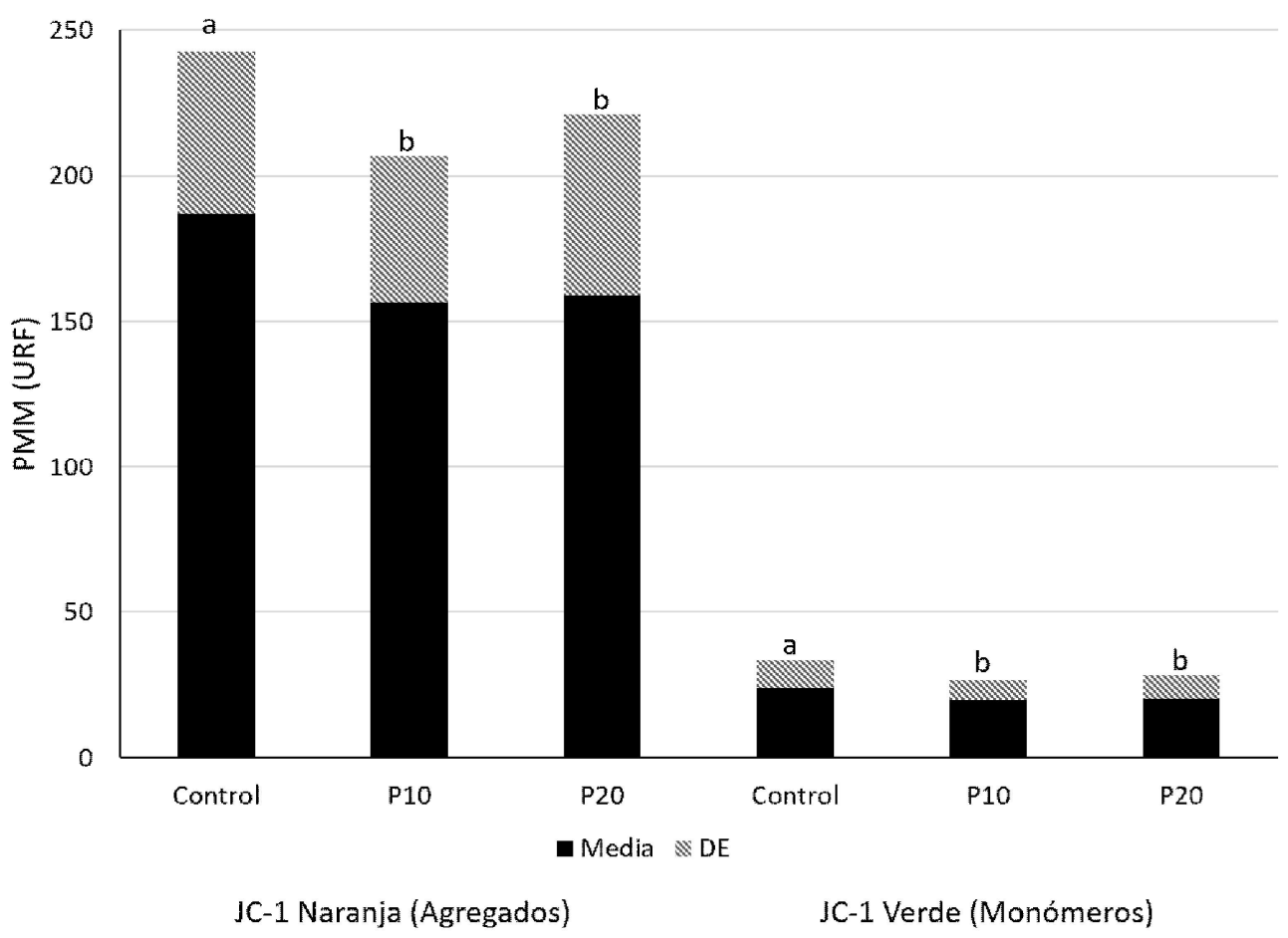

Figura 1. Potencial de membrana mitocondrial posdescongelación de semen equino tratado con dos proporciones de plasma seminal ( $\mathrm{n}=15)$. PMM: potencial de membrana mitocondrial. URF: unidades relativas de fluorescencia. P10: suplementación con 10\% de plasma seminal. P20: suplementación con 20\% de plasma seminal. Letras diferentes indican diferencia estadística entre tratamientos $(p<0.05)$

en la actividad antioxidante del semen equino a través del PS (Wnuk et al., 2010; Usuga et al., 2017).

La disminución de la movilidad del semen equino criopreservado se ha relacionado con la peroxidación lipídica de la membrana plasmática y la alteración de las mitocondrias (Darr et al., 2016). En este estudio se hallaron correlaciones positivas entre los niveles de PMM altos y bajos con la actividad de SOD (0.52 y 0.49), así como con la MOV $(0.22$ y 0.26$)$ y la VIT $(0.28$ y 0.28$)$ de los espermatozoides, respectivamente $(\mathrm{p}<0.05)$. Estos resultados muestran que la modulación de la actividad mitocondrial puede ser importante en la mitigación de los impactos deletéreos de la congelación, toda vez que se conoce que el daño de la membrana plasmática da como resultado la pérdida irreversible de la capacidad de fertilización de los espermatozoides (Brito et al., 2003). Sin embargo, también se encontraron coeficientes de correlación negativos entre los niveles de PMM altos y bajos con la IM (-0.23 y $0.21)$, respectivamente $(\mathrm{p}<0.05)$, lo cual podría estar relacionado con un incremento en la peroxidación lipídica por una mayor producción de ROS (García et al., 2011). Adicionalmente, la acción de la SOD podría ser trascendental en la disminución del estrés oxidativo, toda vez que el anión superóxido $\left(\mathrm{O}_{2}{ }^{-}\right)$parece ser la ERO primaria generada a nivel mitocondrial por los espermatozoides equinos (Ball, 2008; Cocchia et al., 2011). 


\section{Conclusiones}

- El plasma seminal genera un incremento de la capacidad antioxidante total representada por el poder reductor férrico (FRAP) del semen equino diluido para la congelación.

- El plasma seminal produce un incremento de la actividad de catalasa del semen equino diluido para la congelación.

- La suplementación con $20 \%$ de plasma seminal en el semen equino diluido para la congelación mejora la movilidad espermática posdescongelación.

- El uso de plasma seminal para la congelación del semen equino produce una reducción posdescongelación del potencial de membrana mitocondrial de los espermatozoides.

\section{Literatura Citada}

1. Aebi H. 1984. Catalase in vitro. Method Enzymol 105: 121-126. doi: 10.1016/ S0076-6879(84)05016-3

2. Al-Essawe E, Johannisson A, Wulf M, Aurich C, Morrell J. 2018a. Improved cryosurvival of stallion spermatozoa after colloid centrifugation is independent of the addition of seminal plasma. Cryobiology 81: 145-152. doi: 10.1016/ j.cryobiol.2018.01.009

3. Al-Essawe E, Wallgren M, Wulf M, Aurich $C$, Macías-García B, Sjunnesson Y, Morrell J. $2018 b$. Seminal plasma influences the fertilizing potential of cryopreserved stallion sperm. Theriogenology 115: 99-107. doi: 10.1016/j.theriogenology.2018.04.021

4. Ball BA. 2008. Oxidative stress, osmotic stress and apoptosis: impacts on sperm function and preservation in the horse. Anim Reprod Sci 107: 257-267. doi: 10.1016/j.anireprosci.2008.04.014

5. Baumber J, Ball B, Gravance C, Medina V, Davies-Morel M. 2000. The effect of reactive oxygen species on equine sperm motility, viability, acrosomal integrity, mitochondrial membrane potential and membrane lipid peroxidation. J Androl 21: 895-902. doi: 10.1002/ j.1939-4640.2000.tb03420.x

6. Baumber J, Ball BA, Linfor J. 2005. Assessment of the cryopreservation of equine spermatozoa in the presence of enzyme scavengers and antioxidants. Am J Vet Res 66: 772-779. doi: 10.2460/ ajvr.2005.66.772

7. Baumber J, Ball BA, Linfor JJ, Meyers $S A$. 2003. Reactive oxygen species and cryopreservation promote DNA fragmentation in equine sperma-tozoa. J Androl 24: 621-628. doi: 10.1002/ j.1939-4640.2003.tb02714.x

8. Baumber J, Vo A, Sabeur K, Ball B. 2002. Generation of reactive oxygen species by equine neutrophils and their effect on motility of equine spermatozoa. Theriogenology 57: 1025-1033. doi: https:/ /doi.org/10.1016/S0093-691X(01)00710-5

9. Benzie I, Strain J. 1996. The ferric reducing ability of plasma (FRAP) as a measure of «antioxidant power»: the FRAP assay. Anal Biochem 239: 70-76. doi: 10.1006/abio.1996.0292

10. Boe-Hansen G, Rego J, Crisp J, Moura A, Nouwens A, Li Y, Venus B, et al. 2015. Seminal plasma proteins and their relationship with percentage of morphologically normal sperm in 2-yearold Brahman (Bos indicus) bulls. Anim Reprod Sci 162: 20-30. doi: 10.1016/ j.anireprosci.2015.09.003

11. Brito L, Barth A, Bilodeau-Goeseels S, Panich P, Kastelic J. 2003. Comparison of methods to evaluate the plasmalemma of bovine sperm and their relationship with in vitro fertilization rate. Theriogenology 60-8: 1539-1551. doi: https://doi.org/10.1016/S0093691X(03)00174-2

12. Brito L, Greene L, Kelleman A, Knobbe M, Turner R. 2011. Effect of method and clinician on stallion sperm morphology evaluation. Theriogenology 76: 745-750. doi: 10.1016/j.theriogenology.2011.04.007 
13. Brum A, Sabeur K, Ball B. 2008. Apoptotic-like changes in equine spermatozoa separated by densitygradient centrifugation or after cryopreservation. Theriogenology 69: 1041-1055. doi: $10.1016 /$ j.theriogenology.2008.01.014

14. Bucci D, Giaretta E, Spinaci M, Rizzato G, Isani G, Mislei B, Mari G, et al. 2016. Characterization of alkaline phosphatase activity in seminal plasma and in fresh and frozen-thawed stallion spermatozoa. Theriogenology 15: 288295. doi: https://doi.org/10.1016/j.theriogenology.2015.09.007

15. Burnaugh L, Sabeur K, Ball B. 2007. Generation of superoxide anion by equine spermatozoa as detected by dihydroethidium. Theriogenology 67: 580-589. doi: 10.1016/j.therioge-nology.-2006.07.021

16. Bustamante I, Pederzolli C, Sgaravatti A, Gregory R, Dutra C, Jobim M, Mattos R. 2009. Skim milk-egg yolk based semen extender compensates for non-enzymatic antioxidant activity loss during equine semen cryopreservation. Anim Reprod 6: 392-399.

17. Cocchia N, Pasolinia M, Mancini R, Petrazzuolo O, Cristofaro I, Rospane I, Sica A, et al. 2011. Effect of sod (superoxide dismutase) protein supplementation in semen extenders on motility, viability, acrosome status and ERK (extracellular signal-regulated kinase) protein phosphorylation of chilled stallion spermatozoa. Theriogenology 75: 12011210. doi: 10.1016/j.therioge-nology.2010.11.031

18. Contri A, Alessia G, Domenico R, Michele P, Sfirro M, Carluccio A. 2012. Effect of sperm concentration on characteristics of frozen-thawed semen in donkeys. Anim Reprod Sci 136: 7480. doi: 10.1016/j.anireprosci.2012.10.022

19. Darr C, Martorana K, Scanlan T, Meyers S. 2016. The effect of low oxygen during the early phases of sperm freezing in stallions with low progressive motility: can we improve post-thaw motility of stallion sperm? J Equine Vet
Sci 42: 44-51. doi: https://doi.org/10.1016/ j.jevs.2016.03.022

20. Ferrante A, Neild D, Caldevilla M, Carretero M, Vazquez, M, Miragaya M. 2016. Effect of autologous seminal plasma on the capacitation and acrosome reaction of equine sperm. J Equine Vet Sci 43: S70. doi: https://doi.org/10.1016/ j.jevs.2016.06.040

21. García B, Fernández, L, Ferrusola C, Salazar-Sandoval C, Rodríguez A, Martinez H, Tapia J, et al. 2011. Membrane lipids of the stallion spermatozoon in relation to sperm quality and susceptibility to lipid peroxidation. Reprod Domest Anim 46: 141-148. doi: 10.1111/j.1439-0531.2010.01609.x

22. Gravance C, Garner D, Baumber J, Ball B. 2000. Assessment of equine sperm mitochondrial function using JC1. Theriogenology 53: 1691-1703. https:// doi.org/10.1016/S0093-691X(00)00308-3

23. Guasti P, Monteiro G, Papa F. 2012. Componentes do plasma seminal e sua influência sobre a criopreservação e fertilidade de espermatozoides equinos. Vet Zootec 19: 169-180.

24. Isachenko V, Isachenko E, Katkov I, Montag M, Dessole S, Nawroth F, Van Der Ven H. 2004. Cryoprotectant-free cryopreservation of human spermatozoa by vitrification and freezing in vapor: effect on motility, DNA integrity, and fertilization ability. Biol Reprod 71: 11671173. doi: 10.1095/biolreprod.104.028811

25. Kareskoski M, Katila T. 2008. Components of stallion seminal plasma and effects of seminal plasma on sperm longevity. Anim Reprod Sci 107: 249-256. doi: 10.1016/j.anireprosci.2008.04.013

26. Koskinen E, Karlsson M, Reilas T, Sankari S, Esala A, Katila T. 2002. Catalase activity and total protein in fractionated stallion seminal plasma. Theriogenology 58: 337-340. doi: 10.1016/S0093-691X(02)00767-7

27. Moore A, Squires E, Graham J. 2005. Effect of seminal plasma on the cryopreservation of equine spermatozoa. Theriogenology 63:2372-2381. doi: https:/ 
/doi.org/10.1016/j.theriogenology.2004.05.032

28. Morte M, Rodrigues A, Soares D, Rodrigues A, Gamboa $S$, RamalhoSantos J. 2008. The quantification of lipid and protein oxidation in stallion spermatozoa and seminal plasma: seasonal distinctions and correlations with DNA strand breaks, classical seminal parameters and stallion fertility. Anim Reprod Sci 106: 36-47. doi: 10.1016/ j.anireprosci.2007.03.020

29. Neuhauser S, Dorfel S., Handler J. 2015. Dose-dependent effects of homologous seminal plasma on motility and kinematic characteristics of post-thaw stallion epididymal spermatozoa. Andrology 3: 536-543. doi: 10.1111/andr.12003

30. Neild D, Chaves G, Flores M, Mora N, Beconi M, Agüero A. 1999. Hypoosmotic test in equine spermatozoa. Theriogenology 51: 721-727. doi: https:// doi.org/10.1016/S0093-691X(99)00021-7

31. Ortega-Ferrusola C, García B, Gallardo-Bolaños J, GonzálesFernández L, Rodriguez-Martinez $\mathrm{H}$, Tapia J, Peña F. 2009. Apoptotic markers can be used to forecast the freezeability of stallion spermatozoa. Anim Reprod Sci 114: 393-403. doi: 10.1016/j.anireprosci.2008.10.005

32. Ou B, Hampsch-Woodill M, Prior R. 2001. Development and validation of an improved oxygen radical absorbance capacity assay using fluorescein as the fluorescent probe. J Agric Food Chem 49: 4619-4626.

33. Pizarro E, Restrepo G, Echeverry J, Rojano, B. 2013. Efecto del plasma seminal sobre el estado redox del semen equino criopreservado. Rev MVZ Córdoba 18: 3672-3680. doi: https://doi.org/ 10.21897/rmvz. 134

34. Ramires-Neto C, Monteiro GA, Soares R, Pedrazzi R, Dell'aqua J, Papa F, Alvarenga M. 2013a. Effect of removing seminal plasma using a sperm filter on the viability of refrigerated stallion semen. J Equine Vet Sci 33: 40-43. doi: https://doi.org/10.1016/j.jevs.2012.04.008
35. Ramires-Neto C, Monteiro G, Soares $R$, Pedrazzi C, Dell'aqua J, Papa F, Chaves M, et al. 2013b. New seminal plasma removal method for freezing stallion semen. Theriogenology 79: 11201123. doi: $10.1016 /$ j.theriogenology. 2013.01.014.

36. Restrepo G, Zapata A, Rojano B. 2015. Evaluación de la capacidad antioxidante total del plasma seminal equino. Zootecnia Trop 31: 79-87.

37. Restrepo G, Rojano B. 2017. Efecto del isoespintanol y el timol en la actividad antioxidante de semen equino diluido con fines de congelación. Rev Med Vet 35: 149-158. doi: http://dx.doi.org/ 10.19052/mv.4397

38. Rodríguez-Martínez $\mathrm{H}$, Kvist $U$, Ernerudh J, Sanz L, Calvete J. 2011. Seminal plasma proteins: what role do they play? Am J Reprod Immunol 66: 1122. doi: 10.1111/j.1600-0897.2011.01033.x

39. Schober D, Aurich C, Nohl H, Gille L. 2007. Inûuence of cryopreservation on mitochondrial functions in equine spermatozoa. Theriogenology 68: 745754. doi: 10.1016/j.therioge-nology.2007.06.004

40. Tavilani H, Goodarzi M, VaisiRaygani A, Salami S, Hassanzadeh T. 2008. Activity of antioxidant enzymes in seminal plasma and their relationship with lipid peroxidation of spermatozoa. Int Braz J Urol 34: 485-491.

41. Usuga A, Rojano B, Restrepo G. 2017. Effect of seminal plasma components on the quality of fresh and cryopreserved stallion semen. J Equine Vet Sci 58: 103111. doi: http://dx.doi.org/10.1016/ j.jevs.2017.09.005

42. Usuga A, Rojano B, Restrepo G. 2018. Association of the cysteine-rich secretory protein-3 (CRISP-3) and some of its polymorphisms with the quality of cryopreserved stallion semen. Reprod Fertil Dev 30: 563-569. doi: 10.1071/ RD17044

43. Vasconcelos A, Santana M, Santos A, Santero $M$, Lagares $M .2010$. Metabolic evaluation of cooled equine 
spermatozoa. Andrología 42: 106-111. doi: 10.1111/j.1439-0272.2009.00963.x

44. Villa N, Castaño D, Duque P, Ceballos A. 2012. Actividad de la glutatión peroxidasa y la superóxido dismutasa en sangre y plasma seminal en caballos colombia-nos. Rev Colomb Cienc Pecu 25: 64-70.

45. Waheed M, El-Bahr S, Al-Haider A. 2013. Influence of seminal plasma antioxidants and osteopontin on fertility of the Arabian horse. J Equine Vet Sci 33: 705-709. https://doi.org/10.1016/ j.jevs.2012.11.006

46. Wnuk M, Lewinska B, Oklejewicz G, Bartosz M, Tischner M, BugnoPoniewierska M. 2010. Redox status of equine seminal plasma reflects the pattern and magnitude of DNA damage in sperm cells. Theriogenology 74: 16771684. doi: $10.1016 / \mathrm{j}$.theriogenology.2010.07.007 